\title{
Erratum
}

\section{Regioselective, One-Pot Syntheses of Symmetrically and Unsymmetrically Halogenated 2',6'-Bispyrazolylpyridines}

Supratim Basak, Pramiti Hui, Rajadurai Chandrasekar* Synthesis 2009, 4042.

The advance online publication (e-First) version of this article contained typographical errors in the author name Pramiti Hui and in Scheme 3.

These errors have been corrected for both the current online version and the print publication. 